\title{
DIAGNÓSTICO DEL PROCESO ARTESANAL DE PRODUCCIÓN DE HILO DE FIBRA DE ALPACA en Puno, Perú*
}

\author{
BERTHA DíAZ-GARAY* \\ https://orcid.org/0000-0002-8409-3210 \\ Rosa PATRICIA LARIOS-FrANCIA* \\ https://orcid.org/0000-0002-1471-9185 \\ Wilfredo HeRnándEZ GorRITTI* \\ https://orcid.org/0000-0002-6122-4935 \\ Rafael Chávez Ugaz* \\ https://orcid.org/0000-0002-9450-0158 \\ Carlos Alberto Gálvez Zárate* \\ https://orcid.org/0000-0002-7723-234X \\ Universidad de Lima, Perú \\ ANDRÉS CONDORI TICONA* \\ https://orcid.org/0000-0001-5959-3515 \\ Centro de Innovación Tecnológica (CITE) de los Camélidos Sudamericanos Puno, Perú \\ TeOdosio Huanca Mamani ( $\dagger$ \\ https://orcid.org/0000-0001-5881-8671 \\ Instituto Nacional de Innovación Agraria (INIA) Puno, Perú
}

Recibido: 30 de agosto del 2020 / Aprobado: 04 de diciembre del 2020

doi: https://doi.org/10.26439/ing.ind2021.n40.5149

RESUMEN: Esta investigación buscaba conocer el estado actual del proceso artesanal de producción de hilo de fibra de alpaca, de la región de Puno en el Perú. Por tal motivo, se realizó un estudio con una perspectiva de alcance descriptivo y exploratorio, teniendo como unidad de análisis a los alpaqueros, artesanos, productores medianos y algunos

\footnotetext{
*Esta investigación ha sido financiada por el Proyecto Concytec - Banco Mundial "Mejoramiento y Ampliación de los Servicios del Sistema Nacional de Ciencia Tecnología e Innovación Tecnológica" 8682-PE, a través de su unidad ejecutora ProCiencia, C089-2018 Fondecyt-BM-IADT-MU, con el objetivo de diagnosticar el estado de la cadena productiva de la fibra de alpaca de la región de Puno.

** Correos electrónicos: bdiaz@ulima.edu.pe, rlariosf@ulima.edu.pe,Rhernand@ulima.edu.pe, Rchavezu@ulima.edu.pe, Cgalvezz@ulima.edu.pe, contiamaru@gmail.com
} 
directivos del sector textil. Se diseñó una investigación documental para obtener datos cualitativos y cuantitativos sobre la producción del hilado de alpaca. En el 2019 se hizo la investigación de campo para recolectar los datos directamente de las comunidades alpaqueras, obteniendo información sobre los métodos del proceso de producción de hilos de fibra de alpaca. Se concluyó que la fabricación artesanal de hilo de alpaca mantiene, principalmente, un proceso manual, sin embargo, se han incorporado pequeñas máquinas como la abridora y la de hilatura, compuestas de dispositivos mecánicos simples, con la finalidad de reducir el tiempo de producción de la fibra y mejorar la calidad del hilo obtenido.

PALABRAS CLAVE: fibra de alpaca / hilos de alpaca / tejido artesanal / hilado artesanal

\section{A DIAGNOSIS OF THE ARTISANAL PRODUCTION PROCESS OF ALPACA FIBER YARN IN PUNO, PERU}

ABSTRACT: This research aimed to find out the current state of the artisanal production process of alpaca fiber yarn in the department of Puno, Peru. To that end, a descriptive and exploratory study was carried out, having as unit of analysis the alpaqueros (alpaca breeders), artisans, medium-scale producers and some textile managers. A documentary research was conducted to obtain qualitative and quantitative data on the production of alpaca yarn. In 2019 , field research was carried out to collect the data directly from the alpaquero communities, thus obtaining information on the methods of the production process of alpaca fiber yarn. It was concluded that the artisanal production of alpaca yarn mainly maintains a handmade process. However, small machines such as opening machines and spinning machines, which consist of simple mechanical devices, have been incorporated in the production process to reduce the fiber production time and to improve the yarn quality.

KEYWORDS: alpaca fiber / alpaca yarn / artisanal fabric / artisanal yarn 


\section{INTRODUCCIÓN}

La alpaca (Vicugna pacos) es una especie doméstica de la familia de los camélidos sudamericanos, se estima que existen alrededor de 114 mil productores agropecuarios que tienen al menos una alpaca, de los cuales el $99 \%$ se concentra en la región andina del Perú, lo que caracteriza a este grupo de productores es el nivel de pobreza, debido a la magnitud e inseguridad de sus ingresos reales, ya que estos no garantizan su subsistencia (Dirección General de Políticas Agrarias, 2017).

Entre los años de 1994 al 2018, se ha logrado incrementar la producción de alpacas en un $50 \%$, según (Huanca, Apaza y Gonzales, 2007) la producción de camélidos está sustentada en cuatro factores importantes, tres de ellos relacionados con el medio ambiente, como la sanidad, la alimentación y el manejo; y el cuarto relacionado con la biología del animal a través de la genética. Sin embargo, no se conocen indicadores que reflejen, paralelamente, un incremento en la productividad de la fibra de alpaca. En ese sentido, los principales problemas que afectan la producción están asociados a la calidad del hilo y la incipiente innovación tecnológica del proceso productivo de hilado de fibra de alpaca.

Dentro de los aspectos más importantes que afectan la calidad, se asocian los cambios climáticos de la región, los cuales son conocidos como "heladas". Para el año 2019, el Poder Ejecutivo destinó un presupuesto de 230 millones de soles para la protección de los productores pecuarios ubicados en zonas altas del país ante las heladas (Dirección General de Políticas Agrarias, noviembre de 2018).

Otro de los aspectos que afectan la producción pecuaria de alpaca, específicamente, al hilado, es la calidad del mismo, debido a posibles factores como el proceso productivo, la falta de estandarización en los métodos artesanales y la escasa innovación tecnológica de los equipos empleados (Alzamora Montes, 2017; Parodi Núñez, 2011).

Bajo este contexto, tanto en el aspecto climático como en el aspecto productivo, la problemática actual hace necesaria la intervención de todas las instituciones, tanto públicas como privadas, organismos del Estado, universidades y centros de investigación, que puedan contribuir a mejorar y solucionar estos aspectos que afectan uno de los principales sectores de la industria peruana como lo es el sector pecuario de la fibra de alpaca.

\section{ANTECEDENTES}

\subsection{Situación actual de la cadena productiva de la alpaca en el Perú}

Según estimaciones de la Dirección General de Políticas Agrarias (septiembre de 2019), “la población mundial de alpacas en el año 2018 ascendió a más de 6 millones de 
unidades; siendo Perú el de mayor población (71,7\%), seguido de Bolivia (8,6\%), Australia (8,2 \%), Estados Unidos (5,8 \%,), Europa (2,5\%), Canadá (0,9 \%), Nueva Zelanda (0,7 \%), Chile $(0,6 \%)$.

Según el IV Censo Nacional Agropecuario (Instituto Nacional de Estadística e Informática [INEI], 2013), informa que la población de alpacas fue de 3685516 . El 78,9 \% de la producción de alpaca corresponde a la raza Huacaya, esta se caracteriza por tener la cobertura total del cuerpo con un vellón muy denso, fibra pesada y un crecimiento de sus hebras en forma vertical hacia el suelo; mientras que el $12 \%$ corresponde a la raza Suri, cuyo vellón es más sedoso, lacio y de mayor crecimiento (Dirección General de Políticas Agrarias, noviembre de 2018). Se considera que la fibra de la especie Suri es de mejor calidad que la Huacaya. Un porcentaje pequeño de la producción de alpacas (7,2 \%) corresponde a alpacas cruzadas.

Entre las regiones con mayor población de alpacas, destaca la región Puno, con aproximadamente 1459903 alpacas, y, en segundo lugar, se encuentra el Cusco, con una población de 545454 alpacas.

De acuerdo al análisis realizado por la Organización de las Naciones Unidas para la Alimentación y la Agricultura (FAO, por sus siglas en inglés), en la cadena de comercialización de la fibra de alpaca existe una serie de eslabones de intermediación hasta llegar a su destino final (Organización de las Naciones Unidas para la Agricultura y la Alimentación, 2005), es así que la fibra de alpaca obtenida por los pequeños productores es distribuida a través de los alcanzadores, quienes tienen un trato directo con los productores, posterior a ello se encuentran los rescatistas, quienes acopian la fibra de los productores, siguiendo en la cadena, los agentes comerciales, hasta llegar, finalmente, a las empresas comercializadoras.

Por su parte, según el informe del South Africa Mohair Cluster (marzo de 2019), respecto al análisis de la cadena de valor de la alpaca peruana, evidenciaron una estructura compleja que implica una multitud de diferentes actores, la misma que se observa en la figura 1: los alpaqueros, quienes son los responsables de la producción, la esquila, la rotación de pastos y tratamiento veterinarios y genéticos; los alcanzadores y rescatistas, quienes actúan como intermediarios entre el productor y los agentes comerciales, que a su vez venden a las unidades de procesamiento, las cuales están constituidas por las empresas que operan internacionalmente; los artesanos, que en su mayoría son mujeres dedicadas a las actividades del tejido; organismos gubernamentales, responsables de la promoción, coordinación y asistencia técnica a los principales actores y las ONG, quienes ofrecen asistencia técnica a los productores para impulsar las actividades de comercialización. 


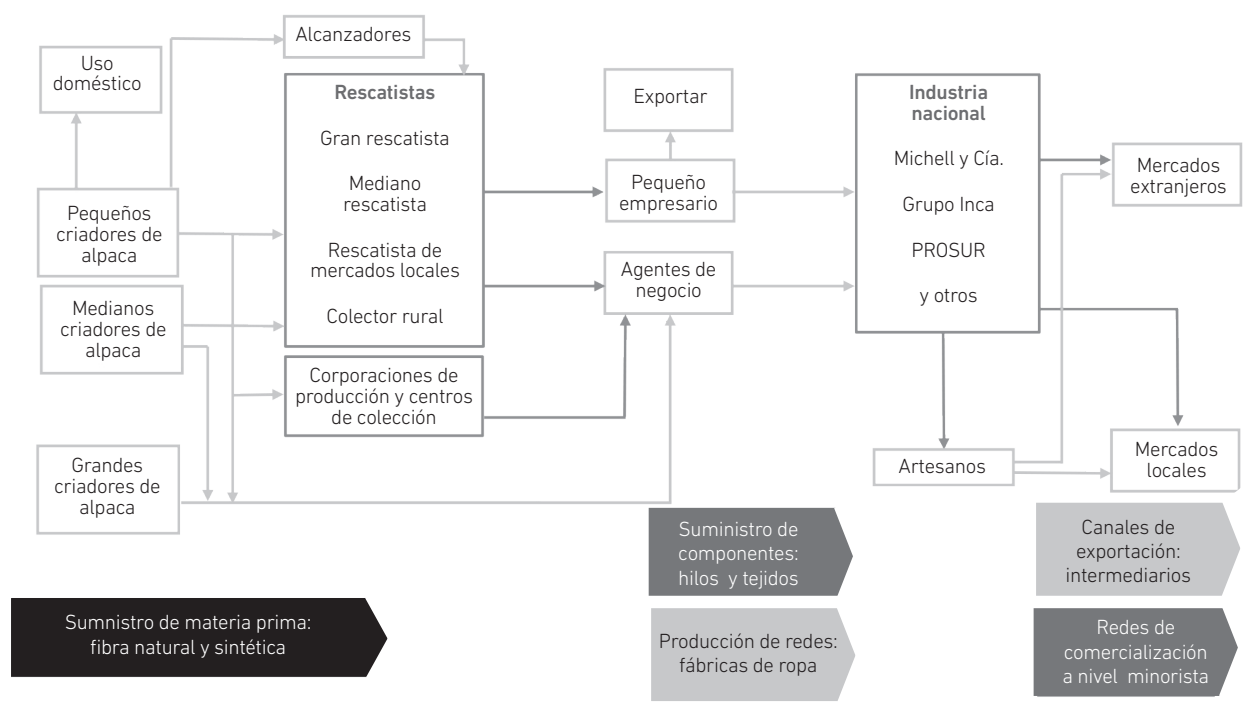

Figura 1. Cadena de valor de producción de la alpaca en el Perú

Fuente: South Africa Mohair Cluster (marzo de 2019)

Con respecto a la producción anual de fibra de alpaca en el Perú, desde el año 2001, la producción se incrementó a una tasa promedio del 1,79 \%. Esto debido al aumento en el número de animales que son esquilados, registrando un incremento de 1,94 a 2,37 millones de unidades (Dirección General de Políticas Agrarias, 2017). Al 2015, la producción de fibra de alpaca alcanzó 4478 toneladas a nivel nacional, siendo la región de Puno la que registró la mayor producción de fibra, 2732 toneladas (tabla 1), aproximadamente, representa el 61 \% del total (Dirección General de Políticas Agrarias, 2017). En el año 2018, la producción de fibra superó las 4600 toneladas (Dirección General de Políticas Agrarias, septiembre de 2019), sin embargo, tal como se muestra en la figura 2 y en la tabla 1, se observa una disminución de la producción promedio de fibra en los últimos siete años, debido a los bajos rendimientos de producción de fibra. Según el Plan Nacional de Desarrollo Ganadero 2017-2027, una de las metas es incrementar el rendimiento de 2,1 al 2021 hasta 2,3 kg/alpaca/año proyectado al 2027 (Dirección General de Políticas Agrarias, 2019).

Según el estudio realizado por la Dirección General de Políticas Agrarias (septiembre de 2019), indica que un aspecto que afecta la producción pecuaria de alpaca es la calidad del hilo y la irregularidad que presentan los productos debido a factores productivos tal como el proceso, la estandarización en el método de hilar artesanal, así como la deficiente innovación tecnológica de los equipos empleados. Así mismo, en el año 2018, el rendimiento promedio de fibra de alpaca fue de 1,9 $\mathrm{kg} / \mathrm{alpaca} / \mathrm{año}$, esta cifra en 
decrecimiento es aún más preocupante si se compara con las ratios de rendimiento en Norte América, que alcanza entre 2,3 a 4,5 kg/alpaca/año, y en Reino Unido, que reporta rendimientos promedios de $2,4 \mathrm{~kg} / \mathrm{alpaca} / \mathrm{año}$, indicando que algunos animales tienen un potencial de 4 a $6 \mathrm{~kg}$ por año.

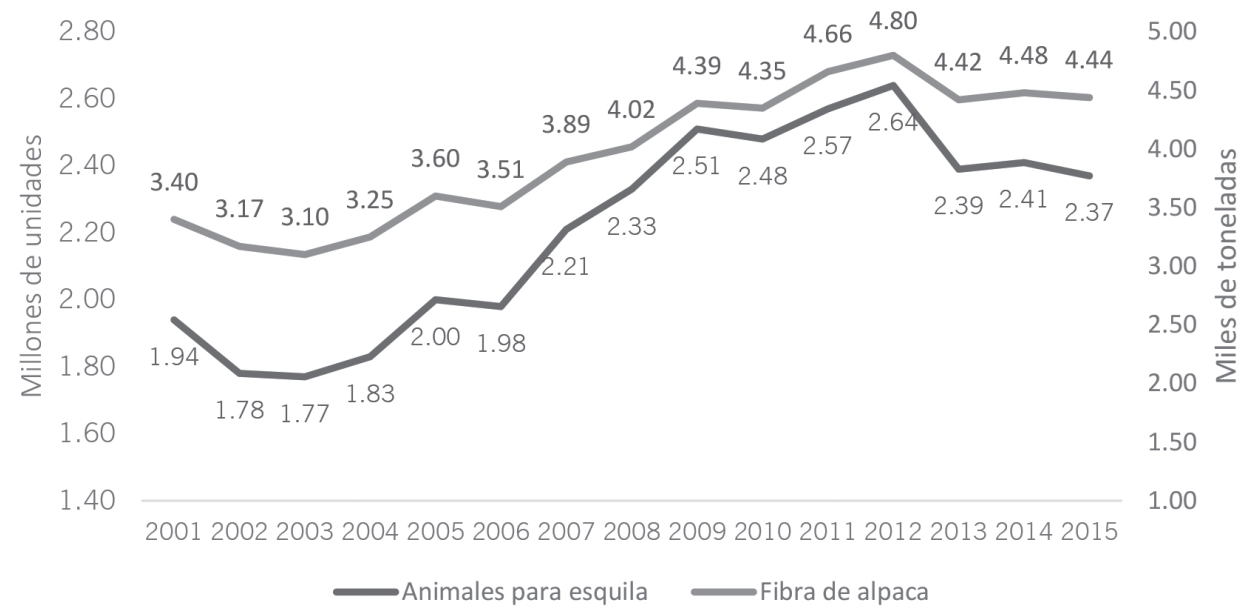

Figura 2. Producción de fibra de alpaca y animales para la esquila

Fuente: Dirección General de Políticas Agrarias (2017)

Tabla 1

Producción de fibra de alpaca en el Perú según región (2012-2018)

\begin{tabular}{lcccccc}
\hline & \multicolumn{7}{c}{ (Toneladas) } & & \\
\cline { 2 - 6 } & 2012 & 2013 & 2014 & 2015 & 2017 & 2018 \\
\hline Puno & 2805 & 2707 & 2764 & 2732 & 2642 & 2644 \\
Cusco & 727 & 583 & 598 & 605 & 569 & 694 \\
Arequipa & 385 & 370 & 415 & 296 & 209 & 186 \\
Ayacucho & 157 & 173 & 139 & 133 & 154 & 180 \\
Pasco & 108 & 130 & 124 & 149 & 162 & 177 \\
Junín & 120 & 102 & 139 & 118 & 141 & 188 \\
Apurímac & 156 & 154 & 147 & 138 & 91 & 81 \\
Huancavelica & 168 & 100 & 69 & 94 & 152 & 164 \\
Moquegua & 98 & 49 & 41 & 76 & 78 & 83 \\
Tacna & 47 & 31 & 26 & 71 & 63 & 119 \\
Lima & 8 & 4 & 4 & 5 & 31 & 35 \\
\hline
\end{tabular}


(continuación)

\begin{tabular}{lcccccc}
\hline La Libertad & 15 & 17 & 17 & 17 & 18 & 17 \\
Huánuco & 1 & 0 & 1 & 2 & 6 & 6 \\
Áncash & 3 & 0 & 0 & 0 & 0 & 0 \\
\hline Total & 4798 & 4420 & 4484 & 4436 & 4316 & 4574 \\
\hline
\end{tabular}

Fuente: Dirección General de Políticas Agrarias (2019)

Otro factor negativo, evidenciado en las prácticas de producción de fibra de alpaca, en el Perú, está relacionado a la conservación genética de la alpaca color que tiene alrededor de 22 colores naturales, que según las preferencias del mercado optan por la fibra de color blanco. Sin embargo, las características de colores naturales cada vez alcanzan un mayor reconocimiento en el mercado internacional, sobre todo en el mercado de lujo y en el consumidor que busca prendas sostenibles (Gallucio, 28 de noviembre de 2018; Rojas Chú, 2016; South Africa Mohair Cluster, marzo de 2019).

\subsection{Proceso productivo artesanal del hilo de fibra de alpaca}

Para describir las características del proceso textil y los métodos que influyen en la producción del hilado, se han observado factores relacionados al estado de tecnificación y automatización, diferenciando del nivel de intensidad manual o artesanal, o una combinación de ambos, cuyos factores influyen en los métodos del proceso de producción del hilado. En ese sentido, se consideran elementos significativos del proceso de producción, la capacidad de detectar defectos u otros problemas, la capacidad de integrar los resultados y parámetros de simulación en la maquinaria textil, así como la fabricación de precisión, herramientas de visualización y diseño de textiles técnicos multifuncionales, o la mejora en la velocidad y el desarrollo flexible de nuevos productos textiles, lo que permite simplificar el proceso de producción, aumentar la tasa de nuevos productos y encontrar nichos de mercado (Bullón, González Arrieta, Hernández Encinas y Dios, 2017).

El proceso textil presenta diferentes tratamientos que se aplican a la fibra de alpaca para su transformación en hilo, el mismo que se puede realizar en forma manual y empleando maquinarias, que en general siguen los procesos que se indican en la figura 3. 


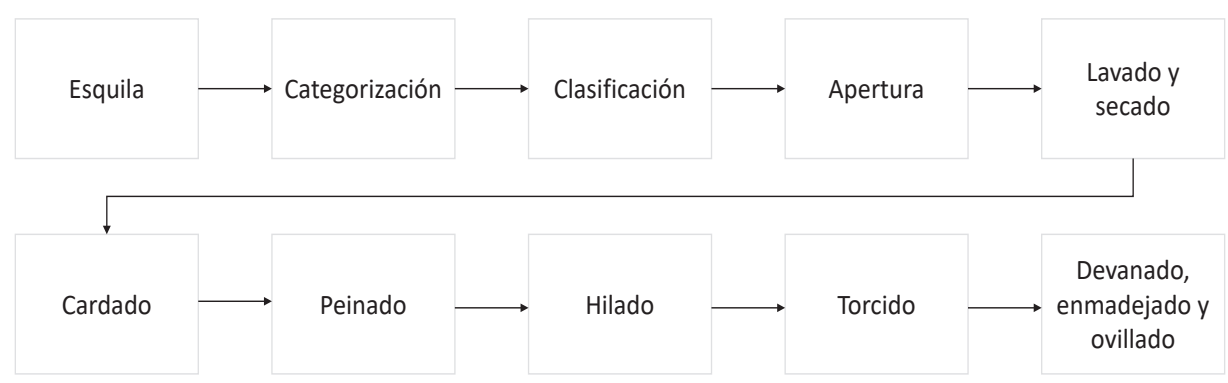

Figura 3. Diagrama de bloques del proceso textil de transformación de la fibra de alpaca Fuente: Terroba Galarreta (mayo de 2017)

La importancia de los métodos tradicionales, la introducción de la tecnología y el logro de la sinergia entre la tecnología y lo artesanal, serán observados durante el proceso textil de la fibra de alpaca, por ello, se establece un parámetro de análisis transversal que permita observar variables que, si bien son propias para el contexto de los Andes peruanos, estas se explican de manera pertinente y sostienen sus prácticas actuales.

\subsubsection{Esquila}

La primera etapa del proceso es la obtención de la fibra y se realiza mediante la esquila, que consiste en cortar y separar el vellón o conjunto total de fibra que cubre a la alpaca (Quispe Peña, Poma Gutiérrez y Purroy Unanua, 2013). La esquila se hace según las normas técnicas peruanas. Las prácticas de esquila a nivel artesanal no varían mucho entre ellas, pues solo se necesitan herramientas básicas como tijeras, cuchillos para el corte del vellón y sogas o el uso de la fuerza humana para evitar movimientos bruscos del animal que puedan conllevar a un corte en su piel (Arciniega Báez, 2013). Según Terroba Galarreta (mayo de 2017), será más eficiente el uso de herramientas eléctricas como cortadoras, a fin de tener mayor número de vellones en menos tiempo; sin embargo, el Instituto Nacional de Innovación Agraria (10 de enero de 2014) indica que luego de realizarse la esquila, debe separarse la braga del vellón para facilitar el clasificado de la fibra.

\subsubsection{Categorización}

Según el Centro de Innovación Tecnológica de Camélidos Sudamericanos Región Puno (2015), la categorización de la fibra de alpaca se realiza por cada vellón esquilado y su calidad se determina en función al porcentaje de fibra fina que se obtenga. Estas categorías son: extrafina, fina, semifina y gruesa. 


\subsubsection{Clasificación de la fibra de alpaca}

En esta etapa del proceso, se realiza la clasificación de fibra de alpaca por raza, color, longitud y finura (Centro de Innovación Tecnológica de Camélidos Sudamericanos Región Puno, 2015).

Cabe resaltar que la mayoría de las empresas realizan este proceso manualmente con la ayuda de personas (por lo general, mujeres) expertas en técnicas visuales que con el uso del tacto permiten diferenciar fácilmente las diferentes finuras de la fibra presentes en las distintas partes del vellón de la alpaca. Existen varias clasificaciones de color, se toma como estándares las siguientes:

Tabla 2

Nomenclatura para variantes de color

\begin{tabular}{lcc}
\hline & Colores enteros & \\
\hline Blanco & Color & Nomenclatura \\
Beige & B \\
Vicuña & LFX \\
Vicuña intenso & LFY \\
Café claro & LFZ \\
Café oscuro marrón & CC \\
Café oscuro negro & COM \\
Gris plata & CON \\
Gris oscuro & GP \\
Negro & & GO \\
\hline & & $\mathrm{N}$ \\
\hline Blanco manchado claro & Colores canosos & \\
Blanco manchado oscuro & & BMC \\
Gris claro & & BMO \\
Negro manchado & GC \\
\hline
\end{tabular}

Colores que pueden tomar diferentes tonalidades

Fuente: Centro de Innovación Tecnológica de Camélidos Sudamericanos Región Puno (2015)

Según Machaca Machaca et al. (2017) y la Norma Técnica Peruana 231.301:2014 Fibra de alpaca clasificada (Instituto Nacional de Calidad, 2014), la clasificación para la finura de la fibra es la siguiente: alpaca súper baby, igual o menor de 20 micrones; alpaca baby, 20,1 a 23 micrones; alpaca fleece, 23,1 a 26,5 micrones; alpaca medium fleece de 
26,6 a 29; huarizo, de 29,1 a 31,5 micrones; gruesa, más de 31,5 micrones, y mixed pieces, fibras cortas superior a los 32 micrones.

La longitud se refiere al largo de la fibra. De acuerdo a lo establecido en la NTP 231.301:2004, la alpaca baby puede llegar a tener una longitud de $65 \mathrm{~mm}$, mientras que la alpaca fleece, alpaca medium y huarizo hasta $70 \mathrm{~mm}$ de longitud. La longitud de la alpaca corta está en el rango de 20 a 50 mm. Schmid (2006) y Rosas Espejo (2012) señalan que, en la práctica, aún existen sistemas de clasificación según la institución que la realice, a pesar de la existencia de normas técnicas. Saldaña Perales (2017) afirma que más del $50 \%$ del vellón de una alpaca corresponde a calidades inferiores (huarizo y gruesas), $35 \%$ a fleece y $8 \%$ a alpaca baby (calidades superiores) y el resto son fibras cortas y mermas (paja, tierra, otros desperdicios, etc.).

\subsubsection{Escarmenado o apertura de la fibra}

Según el Centro de Innovación Tecnológica de Camélidos Sudamericanos Región Puno (2015) y Schmid (2006), en el trabajo artesanal, el escarmenado permite la separación de impurezas como pajas, abono de animal, mechas quemadas por el sol, sustancias insolubles, material mineral y humedad; mientras Saldaña Perales (2017) menciona que la apertura es necesaria para facilitar la carga y realizar un eficiente lavado.

Arciniega Báez (2013) señala dos formas artesanales para realizar el escarmenado, la forma manual en la cual se abre y se extraen los residuos manualmente para obtener la fibra limpia y evitar la formación de los copos o grupos de fibras y disgregarlos; y en la abridora artesanal se evita que la fibra sea maltratada y, además, permite eliminar la mayor cantidad de impurezas para obtener fibra desintegrada mucho más limpia. Por su parte, Aguilar Sánchez (2014) señala que el proceso de apertura debe incluir tres etapas de operación: reclasificado, escarmenado y apertura.

\subsubsection{Lavado y secado}

En el proceso artesanal de lavado, el Centro de Innovación Tecnológica de Camélidos Sudamericanos Región Puno (2015) explica que en esta etapa se eliminan las impurezas, la grasa y la suciedad de la fibra, pero a nivel artesanal el lavado se realiza una vez que se ha obtenido la madeja, se utilizan de 2 a 5 tinas de material plástico, pasando por proceso de lavado con detergente y bicarbonato de sodio y enjuague (Talledo Benites, Chanjí Díaz, Peche Carbonel y Aucahuasi Oviedo, 2017; Terroba Galarreta, mayo de 2017).

Con respecto al secado a nivel artesanal, se usan colgadores, en donde con ayuda del flujo de aire se espera reducir los niveles de humedad (Aguilar Sánchez, 2014). Otros autores señalan la importancia de usar superficies respirables y secadores que generen flujo de aire caliente que permita que el exceso de agua sobre la fibra se evapore. 


\subsubsection{Cardado}

El cardado permitirá convertir la fibra enredada en un "sliver" (fibra cardada) con una mejor paralelización de las fibras. Además, esta operación permite abrir, individualizar mechones de alpaca enredados y desgrasados previamente con una rotura de fibras mínima, mezclar uniformemente fibras de diferentes tipos, eliminar impurezas que causen defectos en la apariencia del hilo y alinear fibras casi paralelamente, evitando daños en la fibra (X. Wang, L. Wang y Liu, 2003). La finalidad es uniformizar el color y la textura de la fibra, y artesanalmente se realiza deslizando o estirando uniformemente la fibra hasta tener "tops", que son fibras preparadas para el hilado que se enroscan u ovillan (Centro de Innovación Tecnológica de Camélidos Sudamericanos Región Puno, 2015).

A nivel artesanal se cuentan con máquinas cardadoras elaboradas a partir de madera, las cuales limitan la producción de la fibra, tal como se comprobó en la visita tecnológica a las comunidades de Wawa Paqocha y se confirmó en Quimsachata (región de Puno) en el año 2019, donde utilizaban una máquina cardadora de madera con dos tambores con púas (uno para acumular la fibra y otro usado como peine); sin embargo, estos no son usados regularmente en el proceso afectando la calidad del mismo (Aguilar Sánchez, 2014), bastará realizar esta operación con una cardadora de madera artesanal.

A nivel industrial y semiindustrial, el cardado permite peinar y unificar la fibra, obteniéndose una mecha uniforme, a través de una serie de rodillos con púas, las cuales peinan la fibra en todo el ancho de la máquina, para posteriormente unirla en una mecha.

\subsubsection{Hilatura}

La hilatura es el proceso mediante el cual se adelgaza la cinta de carda y del tren de estiraje y se realiza la torsión para otorgar resistencia. Posterior a ello, se procede al peinado requerido para obtener un hilo de alta calidad. Como parte del proceso de hilatura, se realiza un subproceso de estirado, que es necesario para dar regularidad a la fibra, enderezándola, ya que la fibra de alpaca es lisa y ondulada.

Los objetivos en esta etapa son: eliminar fibras cortas y muy enredadas, además de descartar materias extrañas (como impurezas), ordenar las fibras largas restantes y ensamblarlas a una cinta continua. El subproducto de esta etapa se denominará "top", el cual es importante para productos finos e hilos de estambre fuertes (X. Wang et al., 2003).

En esta operación se convierte la fibra de alpaca en hilo. Los números que describen las características de un hilo se denominan "título". El título es la relación entre peso y longitud de un hilado $(\mathrm{kg} / \mathrm{km} \circ \mathrm{g} / \mathrm{m})$, por ejemplo, un título $2 / 16$ indica que en $2 \mathrm{~kg}$ se tienen 16 km de hilo (Centro de Innovación Tecnológica de Camélidos Sudamericanos Región Puno, 2015). 


\subsubsection{Torcido}

Se define como la acción y el efecto de dar vueltas sobre su propio eje, por unidades de longitud de un hilo o hebra textil. Al torcer una mecha de fibras estiradas para convertirlas en hilo, se le brinda la resistencia y la elasticidad necesaria para su uso en tejeduría. La torsión es un parámetro de gran importancia en el diseño de los tejidos. Se realiza tomando dos o más hebras de hilo y juntándolas. También se efectúa el retorcido, este proceso comprende torcer en sentido contrario dos o más cabos de hilo torcido. El proceso artesanal se hace de acuerdo con el tipo de tejido a realizar, para tejido de punto deberá ser ligero y para tejido plano debe ser un torcido más rígido.

\subsubsection{Devanado, enmadejado y ovillado}

El devanado es un proceso en el cual se trata de enrollar el hilo sobre un instrumento llamado "cañón" o "bobina". El enmadejado o "aspar" se realiza como proceso artesanal y permite ordenar en forma de madeja para facilitar el lavado y teñido, la cual debe estar bien hecha para no dificultar la formación del ovillo. En varias comunidades, luego del enmadejado, recién se procede al lavado. El ovillado es una operación previa al tejido, en el cual el hilo se envuelve, uniformemente, para una adecuada presentación y fácil tejido (Centro de Innovación Tecnológica de Camélidos Sudamericanos Región Puno, 2015). Se puede también realizar el bobinado, proceso en el cual se elaboran mezclas de diferentes hilos, colores e incluso diferentes títulos.

\section{METODOLOGÍA DE LA INVESTIGACIÓN}

Se realizó un estudio con una perspectiva de alcance descriptivo y exploratorio, utilizando el muestreo discrecional para la selección de individuos, debido a que los encuestados deben ser conocedores del proceso de hilatura, por ello se consideró como unidad de análisis a los alpaqueros, artesanos, productores medianos y algunos directivos del sector textil alpaquero. Para un mejor entendimiento del proceso productivo y con la finalidad de evaluar las oportunidades de mejora que se deberán aplicar, en cada una de las actividades de transformación desde fibra hasta hilo, se diseñó una investigación documental a través de los trabajos previos y material bibliográfico para obtener datos cualitativos y cuantitativos sobre la producción del hilado de alpaca. Se revisó la literatura para conocer los procesos industriales y hacer una comparación con los artesanales y, luego, el año 2019, se hizo una investigación de campo para recolectar los datos, directamente, de las comunidades alpaqueras de la región de Puno.

Como parte del trabajo de campo, se seleccionaron a los dieciséis entrevistados de acuerdo con sus conocimientos, experiencias y habilidades en el proceso de hilatura, materia de estudio. Se organizó un taller donde participaron los diferentes actores del sector alpaquero en la ciudad de Puno, formulándoles las siguientes preguntas: 
- ¿Qué problemas se presentan en la producción artesanal del hilo de alpaca?

- ¿Qué diferencias existen entre la producción artesanal y la producción industrial?

- ¿Qué mejoras consideran ustedes que podrían realizarse en las operaciones del proceso de producción de hilos de alpaca?

Los instrumentos utilizados fueron: cuestionarios de enfoque cuantitativo y cualitativo, para identificar las características de las organizaciones, comunidades u asociaciones, información de producción, así como de mercado. Además, se realizaron entrevistas dirigidas a profesionales del sector textil, autoridades de instituciones del Gobierno y empresarios alpaqueros de la región de Puno. Los participantes de las entrevistas de investigación se mencionan en la tabla 3.

Tabla 3

Lista de entrevistados según su actividad y lugar de trabajo

\begin{tabular}{|c|c|c|}
\hline Nombre & Actividad & Localidad \\
\hline Entrevistado 1 & Médico veterinario del INIA & Puno \\
\hline Entrevistado 2 & Criadora alpaquera & Zona de Santa Lucía \\
\hline Entrevistado 3 & Maestra de hilado artesanal & Provincia de Lampa \\
\hline Entrevistado 4 & Asociación de Artesanas Chuspa de Oro & $\begin{array}{l}\text { Distrito de Vila, provincia de } \\
\text { Lampa }\end{array}$ \\
\hline Entrevistado 5 & Presidenta de la Asociación de Artesanas & Comunidad de Chingana \\
\hline Entrevistado 6 & Alpaquera y facilitadora & Puno \\
\hline Entrevistado 7 & Artesana de hilos y tejidos & Puno \\
\hline Entrevistado 8 & $\begin{array}{l}\text { Fiscal y expresidenta de la Asociación de Artesanas } \\
\text { Chuspa de Oro }\end{array}$ & $\begin{array}{l}\text { Distrito de Vila, provincia de } \\
\text { Lampa }\end{array}$ \\
\hline Entrevistado 9 & Médico veterinario de la Agencia Agraria & Lampa \\
\hline Entrevistado 10 & Criador alpaquero & Puno \\
\hline Entrevistado 11 & $\begin{array}{l}\text { Médico veterinario, director ejecutivo del CITE de los } \\
\text { Camélidos Sudamericanos }\end{array}$ & Puno \\
\hline Entrevistado 12 & Coordinadora del proyecto Tejiendo la Solidaridad & $\begin{array}{l}\text { Corporación Internacional } \\
\text { Italiana / proyecto binacional } \\
\text { Perú-Bolivia }\end{array}$ \\
\hline Entrevistado 13 & Gerente de Operaciones de la Empresa Textilogía S. A. & Huancavelica/Lima \\
\hline Entrevistado 14 & $\begin{array}{l}\text { Encargado del área de producción de la ganadería } \\
\text { Wawa Paqocha }\end{array}$ & Cojata, Puno \\
\hline Entrevistado 15 & $\begin{array}{l}\text { Gerente de la empresa comercializadora Awaq } \\
\text { Pacocha }\end{array}$ & Cojata, Puno \\
\hline Entrevistado 16 & $\begin{array}{l}\text { Director del Centro de Innovación Tecnológica CITE } \\
\text { Textil Camélidos Puno }\end{array}$ & Puno \\
\hline
\end{tabular}

Elaboración propia 


\section{RESULTADOS}

Los hallazgos encontrados permitieron categorizar cada una de las actividades del proceso de producción artesanal de hilo de fibra de alpaca, describiéndose el método empleado, las tecnologías utilizadas, el nivel de uso de mano de obra, los materiales y los recursos nativos, las costumbres y las tradiciones presentes en sus actividades, acceso a técnicas externas, así como el lugar de trabajo.

\subsection{La esquila y categorización de fibra de alpaca}

Los entrevistados manifestaron haber realizado mejoras en la operación de la esquila, producto de las capacitaciones recibidas; la mayoría de las organizaciones de alpaqueros tienen conocimiento acerca del proceso de esquila. Después de la esquila, sigue la categorización, teniendo en cuenta la separación por razas y por colores, con un especial cuidado con el color blanco. Las categorías trabajadas son extrafina, fina, semifina y gruesa. Un aspecto importante en la categorización es la separación del manto y bragas como parte del vellón esquilado.

\subsection{La clasificación y limpieza de la fibra}

De acuerdo con lo mencionado por los entrevistados, la fibra de alpaca es clasificada en calidades: súper baby, baby, fleece, medium $\mathrm{FI}$, huarizo, alpaca gruesa [AG] y alpaca corta [MP]. Algunos de ellos indican que la gran parte de las calidades superiores de la fibra se comercializan para la industria, y las calidades inferiores se utilizan para la producción artesanal, pero de esta calidad de fibra se obtiene poca cantidad.

Nosotros, como asociación, tenemos nuestra alpaca, la esquilamos y clasificamos, lo que es la baby alpaca la usamos para la producción de artesanía. Todas las alpacas no tienen la calidad baby, solo algunas, en especial, alcanzan de 200 a 250 gramos, las que tienen mayor producción llegan hasta 300 gramos. (Entrevistado 2)

Esta etapa es un trabajo que requiere especialización de las personas clasificadoras, que generalmente son mujeres, cuya experiencia es de vital importancia. Es un trabajo que necesita tanto del tacto como de la vista y por la generación del polvillo durante el proceso, los ojos y las vías respiratorias se ven afectados.

Los polvillos finos quedan en la nariz y por eso usamos algodón por dentro, barbijo y coca. También se generan problemas en los ojos, ocasionando disminución de la vista. Algunos usan tapabocas y anteojos, pero no es igual, no te ayudan porque todo el trabajo debe ser al tacto y a la vista. (Entrevistado 6)

Es importante trabajar en el diseño de máscaras, de algún tipo, porque los trabajadores tienen muchos problemas en los ojos, la garganta, la nariz, por el hilo pequeñito que sale, tanto a nivel de las clasificadoras con el acopio y en el caso de las artesanas también. (Entrevistado 12) 
Los entrevistados sugieren incorporar mesas rotatorias para la clasificación con zarandas o con fajas transportadoras, que mejoran la posición del trabajo actual, que se realiza sobre el suelo, y ayudan a disminuir la contaminación de paja, polvo y otros materiales que están adheridos a la fibra.

\subsection{El escarmenado}

Una vez clasificada la fibra se procede al escarmenado, este es un proceso manual, tal como indican los entrevistados, y es de gran importancia para garantizar la uniformidad del color.

El escarmenado es un proceso manual, cuando una fibra no está escarmenada es imposible sacar las bolitas; por eso si se procede a cardar sin realizar el escarmenado, como en el cardado se mezcla todo, entonces, no se logra uniformizar el color. (Entrevistado 3)

Nosotros usamos primero una máquina llamada aperturadora, la cual hace que se eliminen las impurezas en un $90 \%$. Luego, el escarmenado es una limpieza manual, entonces tenemos unas 60 señoras de las comunidades que trabajan en sus casas y con pinzas limpian la fibra manualmente. Después de la aperturadora, el proceso de escarmenado es muy rápido, se puede producir alrededor de 10 kilos de fibra por día. (Entrevistado 13)

\subsection{El cardado}

Los entrevistados manifiestan que, luego de la clasificación, no todos llegan a realizar el cardado, que es la obtención de fibras homogéneas ordenadas en paralelo. Ellos mencionan que faltan equipos, tales como la cardadora, la peinadora y la lavadora. Debido a esto, los productos finales, como las prendas artesanales, no son apreciados por presentar varias tonalidades, pues sin el cardado no se logra la estandarización del hilo.

La limpieza de la fibra es necesaria como lo es también el cardado, pues elimina la suciedad y las pajas. El cardado ayuda a ablandar las fibras, hacerlas más suaves y manipulables para luego hacer el hilado. (Entrevistado 11)

Una de las máquinas indispensables para obtener la calidad del hilado es la cardadora, hay unas manuales; hemos adquirido una que tenemos en Juliaca y es muy útil. (Entrevistado 2)

La cardadora es muy importante en lo que respecta al hilado, porque cuando se hila sin cardar no se uniformizan los colores. Tanto la peinadora como la cardadora son necesarias para sacar la fibra en tops, como lo hacen en las empresas. (Entrevistado 7)

Hay que cardar las fibras para crear una mecha de color sólido para confeccionar una prenda de un solo color. Aunque tengan hilo de calidad, si no usan la cardadora, la prenda se ve rústica y artesanal. (Entrevistado 13) 
Me gustaría que las máquinas funcionaran con energía solar o energía eléctrica, porque las manuales necesitan de dos personas, una que alimenta la fibra y otra que va moviendo, eso quita tiempo. (Entrevistado 14)

\subsection{El lavado}

De acuerdo con la experiencia de los entrevistados, manifiestan que existe una diferencia entre el proceso de lavado que se realiza a nivel artesanal, empleando detergentes sintéticos o naturales, con respecto al lavado de las empresas a nivel industrial: los alpaqueros, normalmente, proceden al lavado, una vez obtenido el hilo, en madeja; a diferencia de las empresas, que lavan en fibra clasificada, porque tienen máquinas grandes y luego realizan el cardado, el peinado para la obtención del top. Existen algunas máquinas artesanales que podrían usarse para el lavado, pero no es una práctica común.

En Lampa elaboran el hilo y luego realizan el lavado, se utilizan los detergentes del mercado, según la capacitación, la mayoría usa jabón líquido. El agua debe estar entre 35 a $37^{\circ} \mathrm{C}$, generalmente, calentamos el agua con cocinas, de leña o gas, pero mayormente se hace con fogón. (Entrevistado 3)

El lavado se realiza a mano con jabón marca Patito y toma un tiempo de 15 minutos por kilo de fibra. (Entrevistado 5)

Se lava con detergente o con champú, para el lavado utilizan agua de manantial cuando están en el campo y de bidón cuando están en el pueblo. (Entrevistado 4)

Son varias etapas de lavado, son diez tinas, en ellas usamos agua tibia. En las dos primeras, el detergente es de uso doméstico, después el champú, luego el enjuague. Se usa también suavizante en el último proceso, así las fibras salen más limpias y, de esta manera, se obtiene fibra sin olor. Para calentar el agua se tienen hornos, quemamos bosta o estiércol, ponemos un cilindro, en un tanque mezclamos agua fría con agua caliente. (Entrevistado 14)

Las empresas medianas no tienen una buena calidad de lavado, nosotros exportamos y los italianos se quejan porque las fibras llegan con mucha grasa. Han realizado análisis de laboratorio y es un problema porque no hay control de calidad. (Entrevistado 12)

\subsection{El hilado}

La mayoría de los productores, en este proceso, trabajan con ruecas, mientras realizan otras actividades en el campo. Además, ellos emplean también pequeñas máquinas alimentadas con arco fotovoltaico con energía solar.

En una hora se hilan 100 gramos, pero depende de la calidad, si es finito toma unas 3 horas; los títulos 12 y 16 se consideran "finito", pero la mayoría no está capacitada en hilado para lograr estos títulos. (Entrevistado 5) 
Nosotros hilamos con rueca, no tenemos máquina de hilar y 100 gramos toma un día. (Entrevistado 4)

La gente aprovecha el tiempo al estar pastando a sus animales $y$, a la vez, pueden estar trabajando, porque en el campo no hay otra manera de distraerse, si no es hilando. Yo diría que un 80 o $90 \%$ de las señoras hilan en el campo y las otras en sus talleres elaboran este hilo artesanal. (Entrevistado 11)

El hilado antes se hacía en ruecas, pero ahora nos hemos equipado con algunas máquinas eléctricas que funcionan con panel solar. En sí nos ayudan en la torsión, ya que no se necesita la rueca. (Entrevistado 14)

Necesitamos una micropeinadora y una máquina hiladora para poder responder al mercado, ya que con la rueca no nos abastece. Nuestro objetivo es ofertar hilo, no solo la fibra de alpaca, esto sería lo mejor para todas las alpaqueras que trabajan conmigo. (Entrevistado 2)

Los productores de Puno reconocen que el trabajo con la rueca es lento, debido a que algunos de ellos han experimentado el uso de pequeñas máquinas de hilatura, pero aún continúan los problemas en su uso y no resuelven algunos detalles del hilado, como la uniformidad del hilo, la graduación de la velocidad para el hilado, la determinación del título del hilo y la posibilidad de recambio del cono.

Las máquinas artesanales actuales permiten que el hilo en el cono se oriente solo hacia una dirección, sería conveniente que se depositara en el cono de forma diagonal, existen algunas máquinas bolivianas con esas características. Muchas veces las mechas tienen un ancho irregular, pero todo el trabajo es manual. Si se quiere más delgado, se hace más delgado, esto facilita el hilado. (Entrevistado 6)

\subsection{El procesamiento de la fibra de alpaca}

Los productores entrevistados que han logrado colocar sus productos a nivel de exportación, tienen una visión global del mercado, por lo que consideran que la rueca no necesariamente sería reemplazada por las máquinas artesanales o industriales. Existe un valor importante en el trabajo manual, que es apreciado por los clientes extranjeros, por ello comentan que se debe mantener este método de producción. Así, también, se valora lo ancestral y el uso de materiales nativos como la alpaca y los tintes naturales.

Es necesario mantener la producción industrial y la artesanal, el cliente lo pide. Siempre estamos capacitando a las comunidades de la región para mejorar sus hilos artesanales. Tenemos maestros artesanos que llevamos a las comunidades para capacitar y llegar a un buen nivel de calidad del hilo, para que se pueda comercializar a nivel internacional, es decir, calidad de exportación. Tenemos ruecas y cardadoras artesanales, que las señoras usan. (Entrevistado 13)

Tenemos el proceso de transformación artesanal de la fibra, en el proceso también podemos hacer el teñido del hilo con hierbas naturales, como el eucalipto o el colli; 
luego hacemos el tejido. Hacemos prendas desde chompas, chalecos, hasta accesorios: chuños, chalinas, manoplas. También vendemos ovillos de hilos, los títulos que sacamos son 10, 12 y hasta 14. (Entrevistado 14)

Existe unanimidad en los entrevistados con referencia a la importancia en empleabilidad que tiene el sector alpaquero en Puno, no solamente con respecto a la crianza y procesamiento de la fibra de alpaca, sino también en el trabajo artesanal de producción de prendas de alpaca.

Lo que queremos es descentralizar todo o hacer una miniplanta artesanal donde se pueda traer la esquila a la zona y poder procesarla aquí. Generalmente, las señoras vienen a hacer prendas aquí, se reúnen cada cierto tiempo, pero no siempre están en la zona. Algunas se van a pastar. Nosotros hacemos los pedidos de unas ciertas cantidades y tipos de productos, así, cada señora la realiza en la zona donde está ubicada, a veces hasta veinte kilómetros a la redonda. No cabe duda, tendríamos la mejor calidad de hilado manejando todo el proceso en un lugar cerrado, donde la fibra no pudiera ser contaminada. (Entrevistado 15)

Existen oportunidades para seguir trabajando y equipar a los productores, hay varios grupos que han trabajado con proyectos de Fondo Empleo, un grupo que es de los Andes, otra cooperativa en Humabamba y otras dos cooperativas que están en Nanay y Andía. También, las señoras de La Serna hacen el producto con el proyecto Fondo Empleo. Eso me permitió equiparlos con una hiladora de rueca que es lo más tradicional que hay, luego hilado a pedal, ya sea de madera o metálico, y otro dispositivo de hilado eléctrico. (Entrevistado 17)

Los entrevistados manifestaron lo importante que es el lugar de trabajo, aunque no se ve como una alternativa de cambio, pues el lugar natural son sus fundos y chacras, ellos también mencionan que Puno es una zona adecuada no solo para la crianza de las alpacas, sino también para el procesamiento de la fibra.

En un lugar como Huancavelica, que es muy alto y extremadamente seco, la falta de humedad genera electricidad estática y durante todos los procesos las máquinas generan mucho estado estático y la fibra se pega a las máquinas y la mecha tiene la tendencia a romperse. En el caso del hilo, ocurre lo mismo, simplemente porque la electricidad atrae la fibra a las máquinas, poniendo mucha presión en la mecha o en el hilo, por eso se rompe, porque es frágil. Por eso tenemos que crear humedad artificial, que podemos hacer, por ejemplo, cerrando todas nuestras puertas y ventanas, prendiendo la lavadora con agua muy caliente para crear humedad ambiental dentro del lugar, pero eso tiene también desventajas de oxidación de las máquinas. Entonces, en lugares como Ayacucho y Huancayo, tienen la altura correcta. (Entrevistado 13)

Con respecto al proceso productivo, se observa que se mantiene el uso de los métodos ancestrales en la crianza de la alpaca, el empleo de materiales nativos y las condiciones del lugar de trabajo, ello es valorado por los entrevistados, quienes consideran que estos aspectos son favorables para la calidad de la fibra de alpaca. En cada 
una de las etapas del proceso productivo, se evidencia un primer nivel de tecnificación, inclusive, en operaciones que, tradicionalmente, han sido netamente manuales.

La tabla 4 presenta el resultado del análisis de la situación actual del proceso productivo de hilatura artesanal en la región de Puno, con respecto a la estructura de los procesos según el nivel de automatización y de intensidad, de acuerdo con el conocimiento artesanal, en relación al proceso de producción encontrado.

Se han identificado dos tipos de procesos, a partir del nivel de uso de los recursos productivos: procesos manuales, en donde el uso de la mano de obra tiene mayor valor en relación al nivel productivo, y procesos automatizados o semiautomatizados, a aquellos cuyo uso de la maquinaria y tecnología proporciona mayor valor o aporta ventajas productivas a nivel del proceso.

En el nivel de tecnificación se ha utilizado una escala que va entre alto, medio o bajo y que considera el tipo de conocimiento especializado, si es que es único y específico, para el tipo de actividad o tareas que se deben realizar en el proceso.

Tabla 4

Factor de tecnificación del proceso de producción del hilado artesanal de la fibra de alpaca

\begin{tabular}{lccc}
\hline \multicolumn{1}{c}{ Proceso } & $\begin{array}{c}\text { Nivel de } \\
\text { automatización }\end{array}$ & $\begin{array}{c}\text { Nivel del tipo de } \\
\text { proceso }\end{array}$ & Tecnificación \\
\hline $\begin{array}{l}\text { Categorización } \\
\text { Clasificación de la fibra } \\
\text { de alpaca }\end{array}$ & Manual & Intensivo & Alta \\
$\begin{array}{l}\text { Escarmenado o apertura } \\
\text { de la fibra }\end{array}$ & Semiautomático & Medio & Baja \\
Lavado y secado & Manual & Medio & Baja \\
Cardado & Semiautomático & Medio & Alta \\
Peinado & Semiautomático & Medio & Media \\
Hilado & Semiautomático & Medio & Media \\
Torcido & Semiautomático & Bajo & Media \\
Devanado & Semiautomático & Bajo & Baja \\
Enmadejado y ovillado & Manual & Bajo & Baja \\
\hline
\end{tabular}

\section{Elaboración propia}

Nota. En el nivel del tipo de proceso se evaluó comparativamente el nivel de intensidad del uso del recurso (mano de obra y maquinaria) en las comunidades observadas. Para determinar el nivel de uso del recurso, se consideraron los resultados de las entrevistas y las apreciaciones de los investigadores efectuadas en el trabajo de campo teniendo en cuenta el impacto del recurso productivo en el proceso observado. 
Las mediciones realizadas (nivel de automatización y nivel de tipo de proceso) para establecer las calificaciones del factor de tecnificación del proceso de producción del hilado de fibra de alpaca artesanal, se hicieron a nivel cualitativo, estableciendo una escala apreciativa (ver figura 4) de los procesos observados, en las comunidades alpaqueras visitadas en Puno, y a partir de las fuentes primarias obtenidas en las entrevistas realizadas en el trabajo de campo, con los productores miembros de las organizaciones vinculadas.

Otro aspecto por considerar es la ausencia de conocimiento tecnológico y científico en las etapas del proceso de producción, observando un uso limitado en las prácticas cotidianas de los productores, desde las actividades de categorización y clasificación de fibra, los de preproducción hasta el proceso de hilatura de fibra de alpaca, obteniéndose madejas u ovillos de hilado artesanal.

Sin embargo, no se puede dejar de resaltar el alto valor histórico que existe en cada actividad artesanal, actividades llenas de cultura, que se van heredando de generación en generación, técnicas del manejo de las fibras de los camélidos que datan de 2500 a. C. para el uso en el desarrollo de textiles. Esto sumado a la riqueza natural de tener alpacas en cerca de 22 colores naturales, los mismos que, como indican Huanca et al., (2007), son de vital importancia trabajar en investigaciones para su conservación, ante en blanqueo inminente de rebaños.

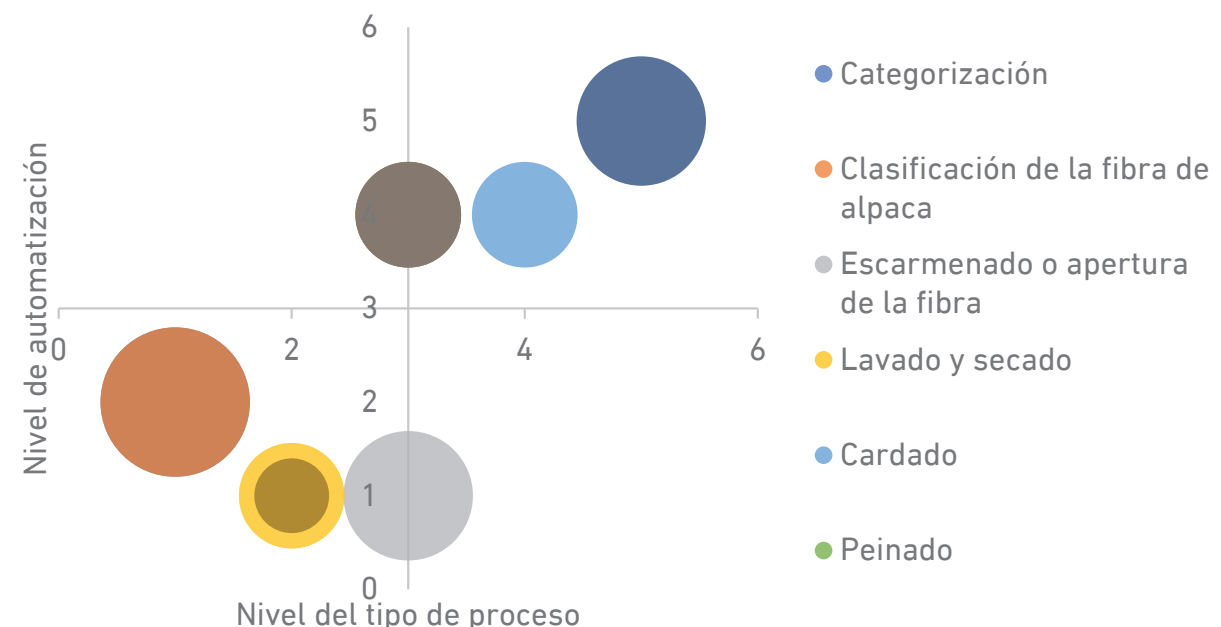

Figura 4. Relación del nivel de automatización y técnica de los procesos de producción de hilado artesanal

\section{Elaboración propia}

Nota. Las observaciones han sido tomadas en una escala del 1 al 5, siendo 1 el menor grado y 5 el mayor grado de relación entre el tipo de proceso y el nivel de automatización. 


\section{CONCLUSIONES/DISCUSIÓN}

El proceso artesanal de producción de hilos de fibra de alpaca ha evolucionado en el tiempo, aun cuando se mantiene, principalmente, un proceso manual, se utilizan pequeñas máquinas artesanales como la abridora y las de hilatura, que reducen el tiempo de producción y han permitido algunas mejoras significativas en el hilo obtenido. La clasificación y la limpieza de la fibra es muy importante para la calidad final del hilo de fibra de alpaca. La mayor parte de la fibra de calidad superior se destina para la venta a la gran industria, mientras que la fibra de calidad inferior se destina para el trabajo artesanal, de esta calidad de fibra se obtiene poca cantidad.

Se identificaron las siguientes diferencias entre el proceso productivo artesanal e industrial de la fibra de alpaca:

- La clasificación de la fibra se realiza a mano por personas expertas, los productores indican que han probado el uso de mesas con zaranda para la clasificación, pero no con buenos resultados. Además, es necesario tomar atención a las condiciones de salud en este trabajo, pues en esta operación se genera un polvillo que causa daño a la vista y a las vías respiratorias.

- La operación de cardado es muy importante para la homogeneización del color, aun cuando se utilice fibra de buena calidad, si no se usa una cardadora, el producto final tendrá tonalidades diferentes. Sin embargo, solo algunos productores realizan el cardado porque no todos cuentan con la maquinaria necesaria.

- La hilatura se realiza en su mayoría con ruecas, con el método ancestral, se utilizan también pequeñas máquinas portátiles que llevan al campo para poder hilar mientras las trabajadoras están pastando su ganado. Se requiere un prototipo de máquina portátil que utilice energía a partir de los paneles solares y también que pueda funcionar con energía eléctrica, este nuevo prototipo debería graduarse para producir el hilo de diferente finura, eso sería un gran aporte para la uniformidad del hilo.

- El lavado se realiza a mano con detergente o champú, se tiene preferencia por el jabón común que se usa para el lavado de ropa, se lava con agua potable que es calentada con leña o bosta. La operación de lavado tiene varias etapas para lo cual se utilizan tinas y el agua utilizada puede ser de manantial o de bidón. En el proceso artesanal, la operación de lavado se realiza en madejas después de la operación de hilatura, a diferencia del proceso industrial que realiza el lavado después de la apertura.

- En la producción artesanal no se realizan las operaciones de peinado y devanado, los artesanos mencionan que no tienen las máquinas para realizar esta operación y manifiestan que esto podría encarecer su producto. 
- Para el enmadejado y ovillado, se emplean pequeños dispositivos llamados ovilladores que ayudan a elaborar las madejas en los tamaños y cantidades necesarios.

El nivel de contribución tecnológico, en cada etapa del proceso productivo, se pueden describir con niveles de gradualidad o incidencia mayor o menor, relacionando el método.

Existen oportunidades de mejora en el proceso productivo artesanal de hilo de fibra de alpaca, particularmente, en la caracterización y clasificación, donde las condiciones de seguridad y salud del trabajador no son las adecuadas. Por otro lado, no todos los artesanos o asociaciones llevan a cabo el proceso de apertura de fibra, lo que dificulta y reduce la posibilidad de generar un hilo de mejor uniformidad; en el proceso de cardado, al no poseer la tecnología adecuada, la calidad del velo resultante aún contiene irregularidades y fibras no paralelizadas; así mismo, los procesos artesanales actuales no permiten la producción de uno de los subproductos más importantes en la industria de alpaca, como es el "top", el cual podría ser comercializado por las comunidades y asociaciones para la elaboración de mezclas u otros artículos textiles. Por ello, al no contar con la maquinaria adecuada trae como consecuencia un volumen reducido de producción. Por último, en el proceso de hilatura, el uso de la rueca mecánica no les permite obtener un hilo uniforme. El proceso de producción de hilo de fibra de alpaca, en cada una de sus etapas, involucra un intensivo uso de mano de obra, incrementando así el tiempo en cada operación, lo que retarda y encarece el proceso.

\section{REFERENCIAS}

Aguilar Sánchez, Y. del C. (2014). Estudio de prefactibilidad para la instalación de una planta procesadora de hilos de fibra de alpaca en la provincia de Lucanas, región Ayacucho (tesis de grado). Universidad Nacional de San Cristóbal de Huamanga. Recuperada de http://repositorio.unsch.edu.pe/handle/UNSCH/934

Alzamora Montes, M. D. (2017). Limitaciones en la producción de fibra de alpaca con valor agregado de los productores de puno (tesis de grado). Universidad San Martín de Porres. Recuperada de https://hdl.handle.net/20.500.12727/2739

Arciniega Báez, S. S. (2013). Mejoramiento de la productividad en la hilatura manual de fibra de alpaca en la comunidad Morochos-Cuycocha-Cotacachi (tesis de grado). Universidad Técnica del Norte, Ibarra, Ecuador. Recuperada de http://repositorio. utn.edu.ec/handle/123456789/1975

Bullón, J., González Arrieta, A., Hernández Encinas, A., y Dios, A. Q. (2017). Manufacturing processes in the textile industry. Expert systems for fabrics production. ADCAIJ: Advances in Distributed Computing and Artificial Intelligence Journal, 6(1), 41-50. http://dx.doi.org/10.14201/ADCAIJ2017614150 
Centro de Innovación Tecnológica de Camélidos Sudamericanos Región Puno. (2015). Hilado artesanal usando energías renovables. https://infohub.practicalaction.org/ bitstream/11283/622414/1/Hilado_artesanal_usando_energias_renovables.pdf

Dirección General de Políticas Agrarias. (2017). Diagnóstico de crianzas priorizadas para el Plan Ganadero 2017-2021. Lima: Ministerio de Agricultura y Riego. Recuperado de http://repositorio.minagri.gob.pe/jspui/bitstream/MINAGRI/328/1/planganadero-2017-2021.pdf

Dirección General de Políticas Agrarias. (noviembre de 2018). Situación de la alpaca en el Perú. Boletín Sumaq Alpaca. Ministerio de Agricultura y Riego. Recuperado de http://agroaldia.minagri.gob.pe/biblioteca/download/pdf/tematicas/l-ciencia/ 101/situacion_alpaca_nov18.pdf

Dirección General de Políticas Agrarias. (septiembre de 2019). Potencial productivo y comercial de la alpaca. Ministerio de Agricultura y Riego. Recuperado de http:// repositorio.midagri.gob.pe:80/jspui/handle/MINAGRI/350

Dirección General de Políticas Agrarias. (2019). Potencial productivo y comercial de la Alpaca. Lima: Ministerio de Agricultura y Riego. Recuperado de https://cdn.www. gob.pe/uploads/document/file/423423/potencial_productivo_comercial_de_ la_alpaca.pdf

Gallucio, A. (28 de noviembre de 2018). Mercado de prendas en alpaca para Europa y EE. UU. Seminario Miércoles del Exportador. Lima: PromPerú. Recuperado de https:// repositorio.promperu.gob.pe/handle/123456789/3557

Huanca, T., Apaza, N., y Gonzáles, M. (2007). Experiencia del INIA en el fortalecimiento del banco de germoplasma de camélidos domésticos. Arc. Latinoam. Prod. Anim, 15(1), 186-194.

Instituto Nacional de Calidad. (2014). Norma Técnica Peruana NTP 231.301:2014 - Fibra de alpaca clasificada. Definiciones, clasificación por grupos de calidades, requisitos y rotulado. Lima, Perú.

Instituto Nacional de Estadística e Informática. (2013). IV Censo Nacional Agropecuario. Resultados Definitivos. IV Censo Nacional Agropecuario 2012. Lima: Ministerio de Agricultura y Riego. Recuperado de http://proyectos.inei.gob.pe/web/ DocumentosPublicos/ResultadosFinalesIVCENAGRO.pdf

Instituto Nacional de Innovación Agraria. (10 de enero de 2014). Transformación artesanal de fibra de alpaca [archivo de video]. Recuperado de https://www.youtube.com/ watch? $=$ Tr3pJTo-QV4

Machaca Machaca, V., Bustinza Choque, A., Corredor Arizapana, F., Paucara Ocsa, V., Quispe Peña, E., y Machaca Machaca, R. (2017). Características de la fibra de alpaca 
Huacaya de Cotaruse, Apurímac, Perú. Revista de Investigaciones Veterinarias del Perú, 28(4), 843-851. http://dx.doi.org/10.15381/rivep.v28i4.13889

Organización de las Naciones Unidas para la Agricultura y la Alimentación. (2005). Situación actual de los camélidos sudamericanos en Perú. Proyecto de cooperación técnica en apoyo a la crianza y aprovechamiento de los camélidos sudamericanos en la región Andina TCP/RLA/2914.

Parodi Núñez, M. N. (2011). Principales problemas en la calidad de la fibra de alpaca que limitan la comercialización de prendas de vestir en el mercado francés. San Martín Emprendedor, Revista de Ciencias Empresariales de la Universidad de San Martín de Porres, 2(2), 21-29.

Quispe Peña, E.,Poma Gutiérrez, A., y Purroy Unanua, A. (2013).Características productivas y textiles de la fibra de alpacas de raza huacaya. Revista Complutense de Ciencias Veterinarias, 7(1), 1-29. https://doi.org/10.5209/rev_RCCV.2013.v7.n1.41413

Rojas Chú, Y. (2016). Alpaca del Perú: Diagnóstico sectorial línea de alpaca. PromPerú. Recuperadodehttp://www.bcrp.gob.pe/docs/Proyeccion-Institucional/Encuentros -Regionales/2016/arequipa/eer-arequipa-2016-rojas.pdf

Rosas Espejo, A. I. (2012). Estudio de las principales características de la fibra de alpaca grasienta y de las condiciones de su proceso de lavado (tesis de grado), Universidad Nacional de Ingeniería. Recuperada de http://cybertesis.uni.edu.pe/handle/ uni/169

Saldaña Perales, L. N. (2017). Categorización, clasificación y procesamiento industrial de la fibra de alpaca (trabajo monográfico para optar el título de ingeniero zootecnista). Universidad Nacional Agraria La Molina. Recuperado de http://repositorio. lamolina.edu.pe/handle/UNALM/3428

Schmid, S. (2006). The value chain of alpaca fiber in Peru, an economic analysis (tesis de maestría). Swiss Federal Institute of Technology Zurich. Recuperada de https:// core.ac.uk/download/pdf/48032051.pdf

South Africa Mohair Cluster. (marzo de 2019). Alpaca Industry of Peru. Recuperado de http://samohaircluster.co.za/wp-content/uploads/2019/07/SAMC-PeruvianAlpaca-Industry-Research-07.03.19.pdf

Talledo Benites, J. C., Chanjí Díaz, E. del R., Peche Carbonel, O. E., y Aucahuasi Oviedo, J. C. (2017). Proyecto de industrialización del hilado de fibra de alpaca de la empresa Nina Pitay SAC (tesis de grado). Universidad San Ignacio de Loyola. Recuperada de http://repositorio.usil.edu.pe/handle/USIL/2987 
Terroba Galarreta, J. F. (mayo de 2017). Implementación de una planta de procesamiento y comercialización de tops e hilados con fibra de alpaca para organizaciones de pequeños y medianos productores. Lima: Perú Agrovet E.I.R.L.

Wang, X., Wang, L., y Liu, X. (2003). The quality and processing performance of alpaca fibres. Rural Industries Research and Development Corporation. Recuperado de https://www.agrifutures.com.au/wp-content/uploads/publications/03-128.pdf 